\title{
OPTICAI INFORMATION ON MASS-LOSS FROM EVOLVED STARS
}

D. Reimers

Institut für Theoretische Physik und Sternwarte

der Universität kiel

\section{Incidence of Observed Mass-Loss in the HR-Diagram.}

Mass-1oss from red-giant stars has been proved by optical observations of violet displaced absorption cores in strong metal lines of low excitation (Deutsch, 1956) long before vast circumstellar shells around the most luminous stars were detected by infrared radiation from circumstellar dust (Gillet et al. 1968, woolf and Ney, 1969), by intrinsic polarization of star light (e.g., serkowski, 1966) and by molecular microwave emission (Wilson and Barrett, 1972). Hot expanding coronae which emit UV lines of highly ionized metals were detected only recently in $\mathrm{G}$ and $\mathrm{K}$ giants (e.g., B Gem, Gerola et al., 1974).

Even today, however, circumstellar absorption lines are both the most sensitive indicators of mass-loss in red giants and the only reliable source of mass-loss rates. This may explain the renewed interest in the study of circumstellar lines in the optical spectra of red giants in the last few years.

A quick glance at a HR-diagram with stars having CS lines (Fig. 1) shows - malnly through optical data - that probably all red giants lose mass.

Mass-loss in very metal deficient red giants has been observed in the brightest giants of $\mathrm{Ml} 3$ and $\mathrm{M} 92$ (Cohen, 1975) and in HD 221 170 (Reimers, 1975 a).

Optical observations also prove that the gas visible through CS lines is really lost to the interstellar medium. This proof is based on observations of CS absorption lines in the spectra of near visual companions of red giants ( $\alpha$ Her: Deutsch, 1956; Reimers, 1977c; $\alpha$ Sco: Deutsch (in Struve and Zebergs, 1962); Kudritzki and Reimers, 1977; $\eta$ Gem and Mira (Deutsch, 1960), and by the observation that in spectroscopic binaries among red giants, the radial veloities of CS lines are stationary, which shows that the shells are decoupled from the photospheres of the stars (RR U Min: Deutsch, 1960; $\mu$ U Ma, $\xi$ Cyg: Reimers, 1977 b). 


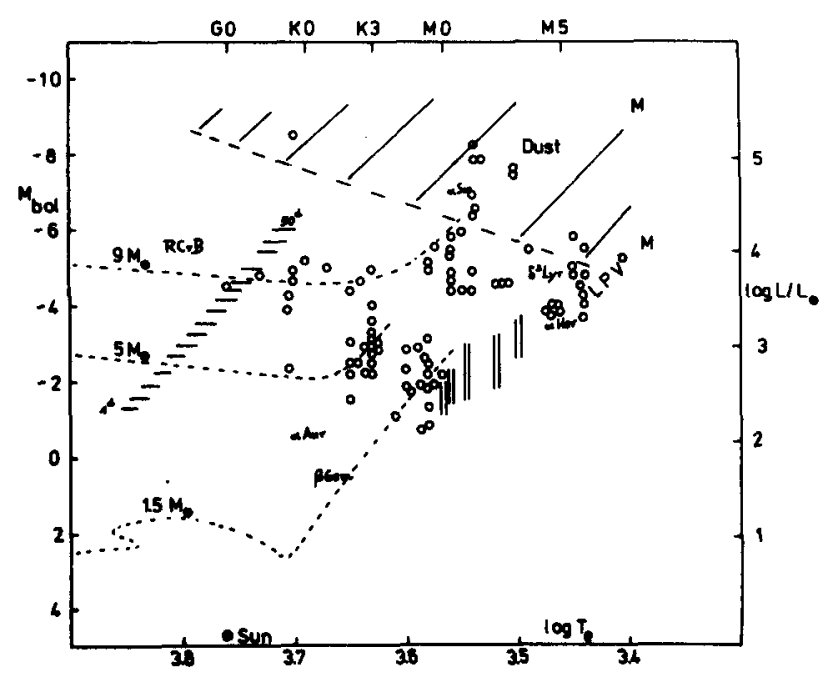

Fig. I Incidence of stars with observed mass-loss in a physical HR diagram (0). Thick vertical bars denote Mo to M4 III stars; $\alpha$ Aur, $\beta$ Gem, and $\alpha$ Agr have hot coronae; stars within the hatched area have dust shells (10- $\mu$ excess); M:stars with molecular microwave emission (Maser); LPV: Mira stars. Evolutionary tracks are by Faulkner (1971) and Faulkner (in Eggen (1974) ).

\section{Appearance of Optical Circumstellar Iines}

The density in cool cs envelopes of red giants is sufficiently low for line scattering to dominate over true absorption. Since the dimensions of the scattering shells are large compared to the stars, the resulting lines have P Cygni like profiles, superimposed upon the underlying strong Fraunhofer lines, if the shell is covered by the spectrograph slit. Weymann (1962) already noticed this on high dispersion photographic spectra of $\alpha$ Ori, while line profiles useful for a quantitative analysis (Fig. 2) became available only recently through high-resolution, double-pass coude spectrographs combined with photoelectric detectors (Goldberg et al. 1975; Bernat and Lambert, 1975; Sanner, 1976).

A nearly complete list of cs lines can be found in Weymann's (1962) work on $\alpha$ Ori, while previous work on velocities and strengths of Cs lines in various types of stars has been reviewed by Deutsch (1960) and Reimers (1975 b). 
The P Cygni type profiles of the lines imply that the expansion velocity of the shell (stellar wind velocity) can be determined reliably only by modelling line profiles by means of spherical line transfer calculations. The position of the absorption core overestimates the wind velocity.

The strong CaII $\mathrm{H}$ and $\mathrm{K}$ cores in supergiants and late $\mathrm{M}$ giants do not seem to show reemission. It appears likely that at short wavelengths and optical depths in the lines of $10^{4}$ to $10^{6}$, the multiple scattered photons are captured by cs dust.

only recently, line reemission from the shells of a Ori at distances up to about 4 arc sec from the star and of $\mu$ Cep ( $\sim 3 "$ ) have been detected by Bernat and Lambert $(1976 \mathrm{~b})$ and Münch et al. (1976) in the $\mathrm{KI} \lambda 7699 \AA$ and $\mathrm{Na} \mathrm{D}_{2}$ lines. The apparent shell diameters correspond to absolute shell radil of $\sim 800 \mathrm{~A} . \mathrm{U}$. and 2000 A.U. respectively. One should consider that if the reemitting shell is resolved on the sky, line profiles depend on completeness of coverage of the shell by the spectrograph slit. Seeing variations then can simulate line profile variations.

At high resolution, the profiles of strong CS lines like NaD and the $\mathrm{KI}$ resonance lines are resolved into multiple velocity components (Goldberg, 1975; Sanner, 1976). In $\mathrm{K}$ giants, $\mathrm{G}$ and $\mathrm{K}$ supergiants, besides the appearance of several components, velocities, strengths and appearance of CS CaII $H$ and $K$ lines - lines can be sharp, diffuse or absent at different times - may vary on a time scale of months (Reimers, 1977 b).

In early M giants, coupled variations of line strengths and velocities of CaII $H$ and $K$ have been observed (Reimers, $1975 \mathrm{a}$ ); in stars like $B$ And and $\delta$ Oph, the sharp violet edge of the lines remains stationary while the red,side moves back and forth. Also, in early M giants, near the visibility limit, the CS CaII $\mathrm{k}$ line may appear complex, i.e., consist of weak multiple velocity components ( $\alpha$ Vul, $\alpha$ Lyn) at high resolution.

\section{III.Stellar Wind Velocities in Red Giants}

As noticed already by Deutsch (1960), the shell expansion velocities of red giants are always much lower than the escape velocities at the stellar surfaces, contrary to what is known from the solar wind or winds in hot stars.

We have measured line shifts in spectra of $\sim 120$ red giants of 
various spectral types and luminosities (Reimers, 1977 b). It appears that there is - in the mean - a continuous transition in proceeding from luminous $M$ supergiants $(10 \mathrm{~km} / \mathrm{s})$ over early $M$ giants $(25 \mathrm{~km} / \mathrm{s})$ and $\mathrm{K}$ giants $(75 \mathrm{~km} / \mathrm{s})$ to the solar wind ( $500 \mathrm{~km} / \mathrm{s})$. This circumstance implies that there is a loose correlation between stellar wind velocities and escape velocities vesc of the form $v_{w} \sim v_{\text {esc }}{ }^{2} \sim g$ R (Fig. $\left.3 a, b\right)$.

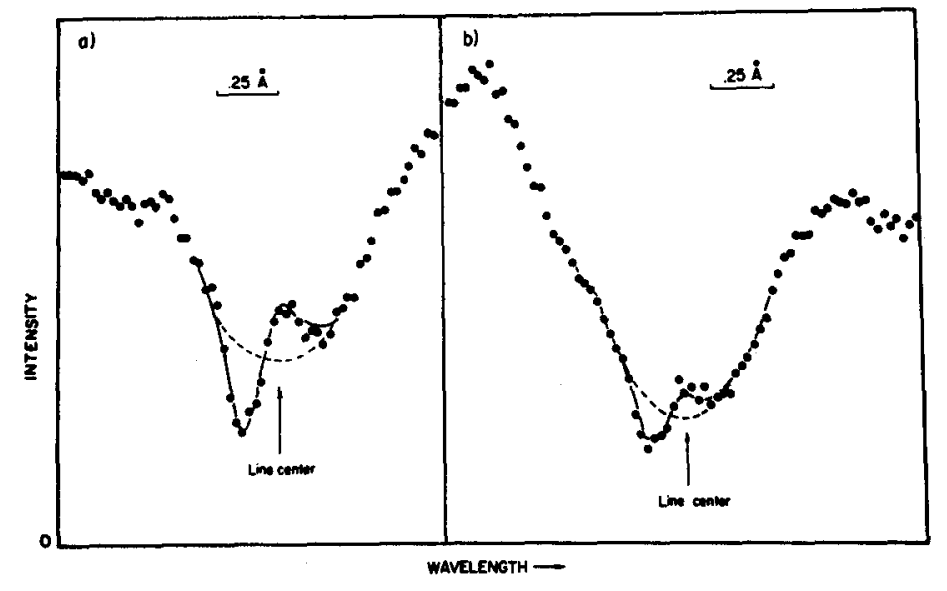

Fig. 2 Circumstellar MnI $\lambda 4030.8 \AA$ and $\lambda 4033.1 \AA$ lines in $\alpha$ Ori with P Cygni profiles, superposed upon the background stellar Fraunhofer lines (Bernat and Lambert, 1976 a).

Violet shifted CaII $\mathrm{k}_{2}$ emission cores reveal that in many red giants the chromospheres are in steady expansion already with nearly half the terminal stellar wind velocities (Fig. 4, from Reimers, 1975 a).

This implies that in many red giants the wind sonic point lies already in the chromospheres of the stars. The expansion of red giant chromospheres can also be seen through blue shifted $\mathrm{H}_{\alpha}$ emission components and - In several $\mathrm{K}$ giants and $\mathrm{G}$ and $\mathrm{K}$ supergiants through a blue shifted HeI $\lambda 10832 \AA$ absorption line (Vaughan and Zirin, 1968). The blue shifts of the HeI line also correspond to typically half the shell expansion velocity.

In several $G$ and $K$ giants and supergiants, observed CaII $H$ and $K$ line shifts vary considerably. Usually, however, cs line shifts in 
M giants and supergiants appear stationary. Since the lines are formed over distances which correspond to flow times of 10 to $10^{3}$ years, stellar wind velocity variations of the order of what is known from the solar wind can only broaden the lines or appear as microturbulence in optically thick lines.

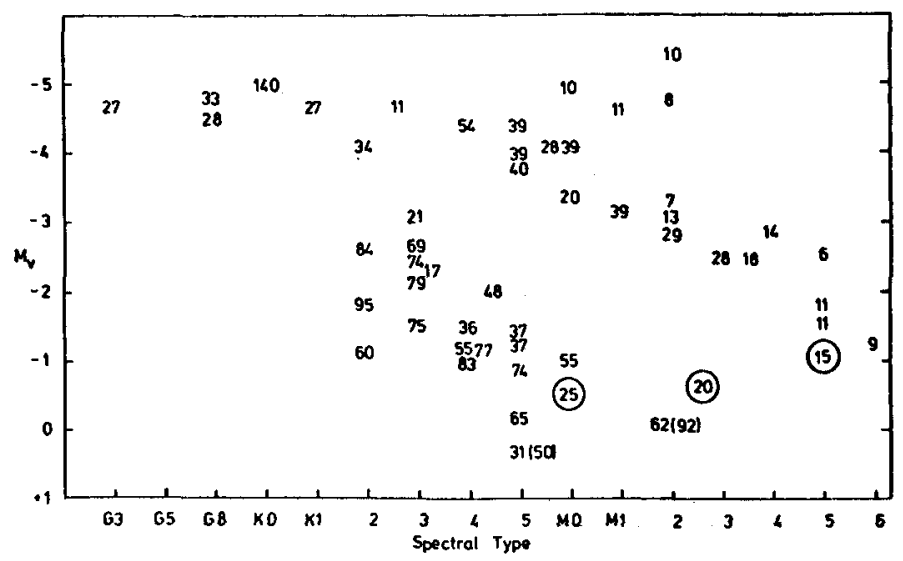

Fig. 3a stellar wind velocities $\left(\mathrm{km} \mathrm{s}^{-1}\right)$ observed in the right-hand upper corner of the HR-diagram (Reimers, 1977 b).

There is also good evidence for velocity gradients in the shells of M giants. In early M giants, the velocity shown by the CaII $\mathrm{K}$ line is about 10 to $15 \mathrm{~km} / \mathrm{s}$ larger than the velocity found from other lines. This difference decreases progressively with spectral type, and $1 \mathrm{n}$ the late $M$ giants and $M$ supergiants, the CaII $K$ velocities are even smaller than those shown by other lines (cf. Fig. 4 and a tentative interpretation in terms of velocity gradients in Reimers (1975 a) ).

\section{Physical Conditions in the Shells}

Most circumstellar lines are resonance lines or arise from low lying levels of neutral and singly ionized metals with excitation potentials less than $\sim \mathrm{leV}$. This circumstance implies that the temperature in the line absorbing region must be low. Weymann (1962) 
deduced a kinetic temperature of the order of $1000 \mathrm{~K}$ from a discussion of the population of fine-structure levels of TiII and FeI. Dust shell temperatures as found e.g., by Gehrz and woolf (1971) are similar.

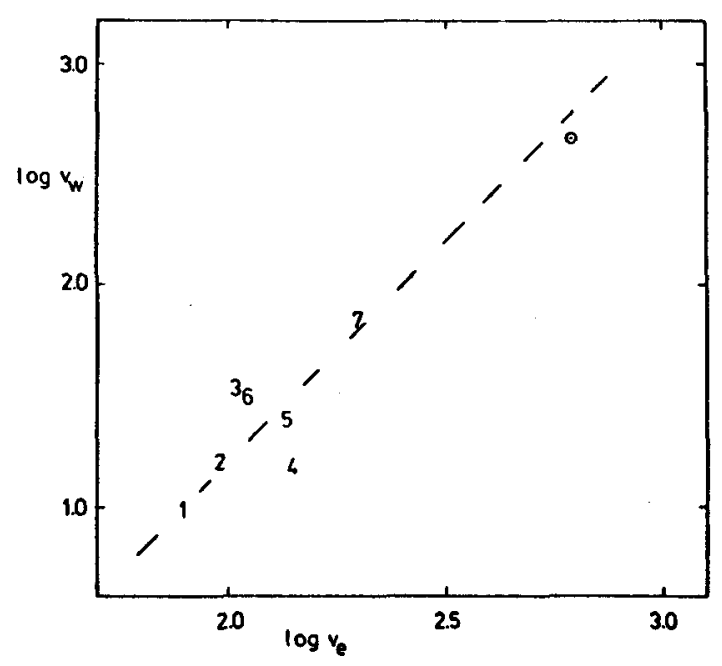

Fig. 3b stellar wind velocity. $v_{w}\left(\mathrm{~km} \mathrm{~s}^{-1}\right)$ versus velocity of escape at the stellar surface $v_{e}\left(\mathrm{~km} \mathrm{~s}^{-1}\right)$ for different groups of red giants (from Fig. 3 a).

Nothing definite can be said about kinetic temperatures in the outer parts of CS envelopes, contrary to what has been claimed by Burgess et al. (1960), Weymann (1962) and Bahcall and wolf (1968) from the absence of lines from excited fine-structure levels of TiII in the spectrum of $\alpha^{2}$ Her. It has been shown that only extreme clumpiness of the matter can be excluded, 1.e., the filling factor must be of the order of unity (Reimers, $1977 \mathrm{c}$ ).

The population of fine-structure levels of TiII observed in the line of sight of $\alpha$ Sco $B$ requires electron collisions at a density $\mathrm{N}_{\mathrm{e}} \sim 10^{3} \mathrm{~cm}^{-3}$ (Kudritzki and Reimers, 1977). Observed column densities of TiII yield a typical hydrogen density $\mathrm{N}_{\mathrm{H}}=10^{3} \mathrm{~cm}^{-3}$ for the line absorbing region ( 500 stellar radii from the $M$ supergiant asco A). Calculations further show that the B star's Strömgren "sphere" in the M star's circumstellar envelope is unlimited in the line of sight of the $B$ star. Therefore, only a negligible fraction 
of $\mathrm{Ti}$ can be condensed into dust and again a filling factor of the or der of unity is required.

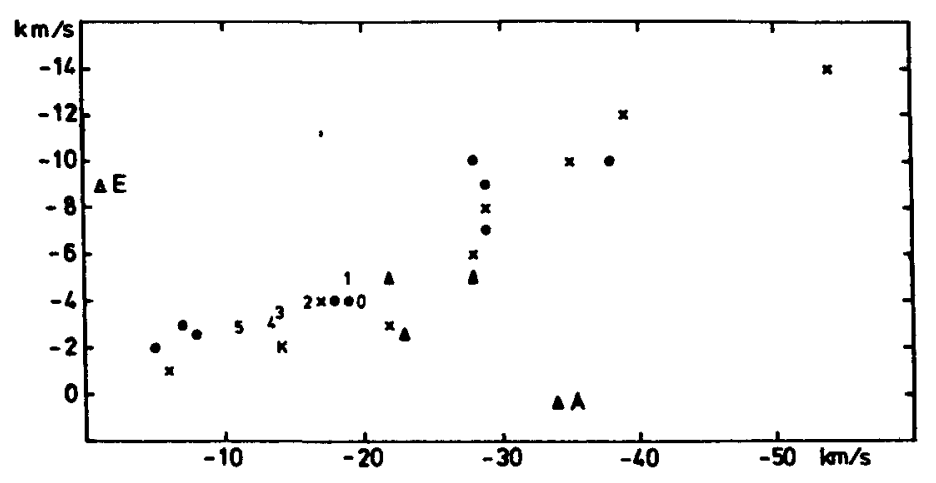

Fig. 4 Circumstellar CaII $K_{3}$ velocity $\Delta A$ versus shift of the chromospheric CaII $\mathrm{K}_{2}^{3}$ emission core $\Delta \mathrm{E}$.Symbols: $0,1, \ldots$ 5: mean values for Mo to M5 III stars ( $\sim 50$ stars); $\mathrm{X}$, and $A: M, K$ and $G$ supergiants (Reimers, $1975 \mathrm{a})$.

Particle densities in the CS shells of red giants are $\sim 10^{7} \mathrm{~cm}^{-3}$ at a typical distance of 5 stellar radii from the stars.

The degree of ionization is generally low in the cool cs envelopes of red giants. Metals with ionization potentials up to that of Fe are mainly singly ionized (cf. Weymann, 1962). This yields a hydrogen to electron density ratio of $\sim 10^{4}$. This is also valid in the outer parts of the envelope of $\alpha$ Her (Reimers, $1977 \mathrm{c}$ ). Possibly there are deviations from statistical equilibrium for ionization since the shell expansion time can be comparable to the recombination time.

\section{Rates of Mass-Loss}

There are several basically different methods for determining mass-loss in red giants from optical observations, namely:

a) the interpretation of the CS line profiles in single stars; Sanner's (1976) paper is the best example of this kind of analysis, 
b) the analysis of CS absorption lines visible in the spectra of near visual companions of red giants like $\alpha^{2}$ Her and a Sco B (Deutsch, 1956; Reimers, $1977 \mathrm{C}$; Kudritzki and Reimers, 1977),

c) radial velocity data from the eclipse of $\zeta$ Aur which suggest a steady expansion of the $K$ supergiant's extended chromosphere (Saito; 1970),

d) violet shifted chromospheric CaII $\mathrm{K}_{2}$ emission cores (cf. Fig. 4) combined with optical depths in the lines in $G$ and $\mathrm{K}$ supergiants (Reimers, 1973); detailed interpretation of $\mathrm{K}_{2}$ emission profiles with help of chromospheric models in $\mathrm{K}$ giants (Chiu et al., 1976).

Methods named under $c)$ and d) allow only rough estimates, and we refer to the original papers.

Let us briefly summarize here how circumstellar p cygni type lines are analyzed in terms of mass-loss following the line of Sanner (1976).

First, the CS line profiles are analyzed with spherical line transfer calculations for column densities of various lons and for kinematics (stellar wind velocity $v_{w}$, random Doppler velocity $v_{d}$, i.e., "microturbulence").

In a second step three distinct types of lines are used to infer different properties of the CS envelopes. Lines of BaII and SrII (dominant ionization stages) are used to obtain hydrogen column densities. The CaII infrared triplet lines, which arise from an excited metastable level populated by the stellar radiation flux, are sensitive to the distance of the shell from the photosphere. The CaII lines have been used first by Weymann (1962) to infer the shell distance. The shell distance, or inner shell radius $R_{1}$, enters linearly into the mass-loss rate. Lines arising from trace ionization states (NaI, K I) are used to obtain the degree of ionization in the envelope. For results, I refer to sanner's (1976) paper in which he analyzed 13 stars. Inner shell radil $R_{i}$ are typically between 2 and 5 stellar radii.

In the following, I shall try to explain why major uncertainties in determining mass-loss rates of single stars pertain to exist although observations and analysts methods have been improved considerably in the last few years. 
1. The separation of CS P Cygni profiles from the cores of strong background Fraunhofer lines on which they are superposed may be difficult. The reasons are insufficient knowledge of the Fraunhofer line profiles (cf. Goldberg, 1975, Fig. 2) and the required strict absence of blends. Observations of a larger number of lines would be helpful. This requires high resolution observations in the wavelength range $\lambda \lambda 3200 \&$ to $4000 \AA$. On the other hand, with shorter wavelengths the increasing cs dust opacity may diminish the reemission, and a guantitative analysis of such "incomplete" P Cygni profiles may become difficult.

2. The inner shell radil $R_{i}$ - transition from extended chromosphere to cool CS envelope - are quite uncertain. Bernat (1977) has even doubted the reality of the cs lines found by Sanner (1976) in the cores of the infrared CaII triplet which have been used to determine $R_{i}$. Moreover, it is questionable whether different lons have the same inner shell radii (e.g., CaII and TiII).

3. The ionization of metals (even of BaII and SrII) is also quite uncertain in the inner parts of the CS shells, because the UV radiation of $M$ giants and supergiants is unknown and due to possible deviations from statistical equilibrium. The only "safe" ion is TilI since 1ts ionization potential is nearly identical with that of hydrogen. Sanner's (1976) electron to hydrogen density ratios $\mathrm{N}_{e} / \mathrm{N}_{\mathrm{H}}$ are therefore questionable. For $\alpha$ Her he found $\sim 3.10^{-7}$, while in the outer parts of the envelope there is at least $\mathrm{N}_{\mathrm{e}} / \mathrm{N}_{\mathrm{H}}=10^{-4}$ (Reimers, $1977 \mathrm{c}$ ).

For both problems - inner shell radil and lonization of metals - a direct solution is not in sight until UV observations, e.g., by the space Telescope, will be available.

4. Further uncertainties arise from the observed multi-component structures (inhomogenities), envelope asymetries (visible through net polarization of star-light), variations of radial velocities of stellar lines which cause variations of the amount of reemission (Goldberg, 1975), and dust in the envelopes (which fraction of a particular element is condensed into dust; opacity).

Uncertainties which affect all methods of mass-loss determination are poorly known luminosities (distances) of $M$ type giants and supergiants and the lack of reliable abundance studies of these stars.

The use of visual binary stars with CS lines in the spectra of both components ( a Her, a Sco) avoids a number of the quoted 
difficulties and, in our opinion, provides the most accurate massloss rates (Reimers, $1977 \mathrm{c}$; Kudritzki and Reimers, 1977). These few accurate mass-loss rates may then be used to establish an independent absolute calibration of relative mass-loss rates (e.g., those of Sanner (1976) and Gehrz' and Woolf's (1971) rates obtained from the infrared'silicate'excess).

As compared with single star CS lines, shell absorption lines visible in the spectra of near visual companions of red supergiants offer the following advantages (details $c$ an be found in the papers on aHer and asco):

- The CS lines are pure absorption lines (like interstellar lines) and may be easily separated from the background stellar spectra: $\alpha^{2}$ Her is a SB, CS lines are stationary; $\alpha$ Sco B is a rapidly rotating $B$ type star, sharp CS lines may be easily recognized.

- For both aHer and aSco, lines of TiII have been used for determining mass-loss of the primaries. The ionization of TiII is negligible in both cases.

- In the case of $\alpha \mathrm{ScO}$, the TiII lines seen in the spectrum of the companion are formed in a HII region. The observed population of the fine-structure levels within the ground state of TiII (relative line strengths), caused by electron collisions, gives a completely independent estimate of the electron density $\mathrm{N}_{e}$ and of the hydrogen density $\mathrm{N}_{\mathrm{H}}$ in the envelope. The corresponding numbers are in full accordance with those derived from absolute TiIr line strengths. The assumptions of solar abundances (no $\mathrm{Ti}$ condensed into dust) and negligible clumpiness of the matter (filling factor $\sim 1$ ) thus appear justified.

- The main advantage is that knowledge of the inner parts of the CS envelopes is not required for determining mass-loss.

The analysis of the secondaries' CS spectra yields mass-loss rates of $2.7 \cdot 10^{-8} \mathrm{M}_{0} / \mathrm{Yr}$ and $1.4 \cdot 10^{-7} \mathrm{M} / \mathrm{Yr}$ for $\alpha^{1_{\mathrm{Her}}}$ and $\alpha$ Sco $\mathrm{A}$. Sanner (1976) found (with $\left.v_{w} / v_{d}=2\right) 5.1 \cdot 10^{-8} \mathrm{M}_{\odot} / y_{r}$ for $\alpha H e r$, which corresponds to $2.2 \cdot 10^{-8} \mathrm{M} / \mathrm{yr}$ for our revised distance of aHer (cf. Reimers, $1977 \mathrm{c}$ ) and a lower limit of $6 \cdot 10^{-8} \mathrm{M} / \mathrm{Yr}$ for $\alpha \mathrm{ScO}$ (without considering the $B$ star's UV radiation which ionizes in part SrII and BaII).

We thus confirm Sanner's (1976) mass-loss rates, while those of Bernat (1977), higher by a factor of $\sim 100$, are erroneous. Sanner's inner shell radii of typically 2 to 5 stellar radil appear approximately correct. 
VI. Dependence of Mass-Loss on Basic Stellar Parameters

Based on optical and infrared data and dimensional arguments, it has been proposed a few years ago that mass-loss depends on stellar parameters luminosity $L$, gravity $g$ and stellar radius $R$ as $\mathrm{dM} / \mathrm{dt}\left(\mathrm{M}_{\odot} / \mathrm{Yr}\right)=\mathrm{c} \cdot \mathrm{L} / \mathrm{g} \cdot \mathrm{R}$ (solar units) with $\mathrm{c}=4 \cdot 10^{-13}$ and an uncertainty of a factor of $\sim 3$ to either side (Fig. 5). Strict validity of this relation would mean that always the same fraction of the stellar luminosity is being used for providing the necessary potential energy of the escaping material.

Sanner's (1976) relative mass-loss rates of 13 red giants are in accordance with the proposed relation.

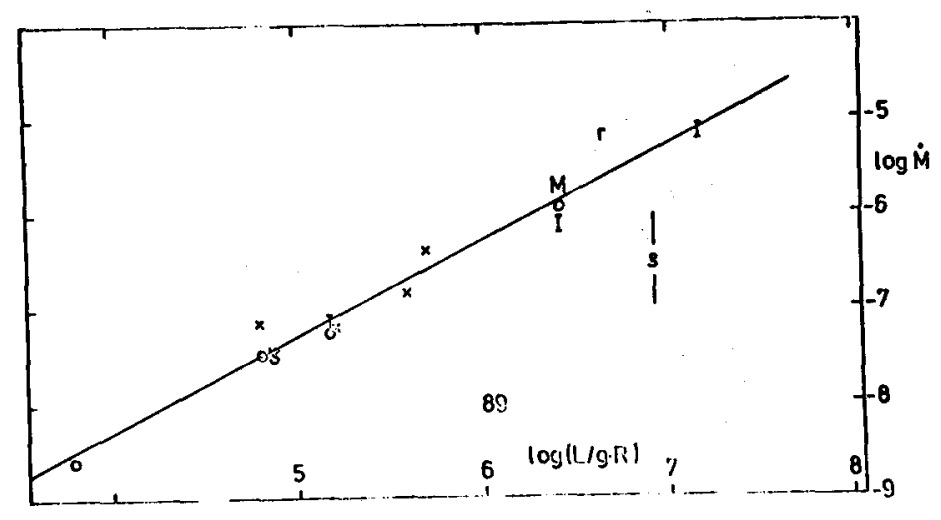

Fig. 5 Mass-loss rates $d M / d t\left(M_{\odot} / Y r\right)$ versus $L / g . R$ (solar units) of various red giants. The solid line represents $\dot{M}=4.10^{-13}$ L/g.R (Reimers, 1975 a).

For establishing a reliable absolute scale of the mass-loss formula, not only accurate mass-loss rates are required. Equally important is knowledge of $\mathrm{L}, \mathrm{R}$, and the mass $M$. Mass and radius cannot be determined with the required accuracy for field stars. Knowledge of the evolutionary stage (1. or 2. giant branch?) is useful too for an understanding of the role of mass-loss in stellar evolution: 
At present we have sufficlently accurate numbers of only 3 stars (Fig. 6): $\alpha^{1}$ Her (mass from comparison of colour-magnitude diagram of the three system members with that of galactic clusters);

a Sco A (member of upper Sco-Cen-association), and $\delta{ }^{2}$ Lyr (cluster member). The only further cluster member which has been observed for mass-loss is the MIII giant HR 3153 in NGC 2516 with $\sim 5$ solar masses (Reimers, 1977 a). Its mass-loss rate is less rellable.

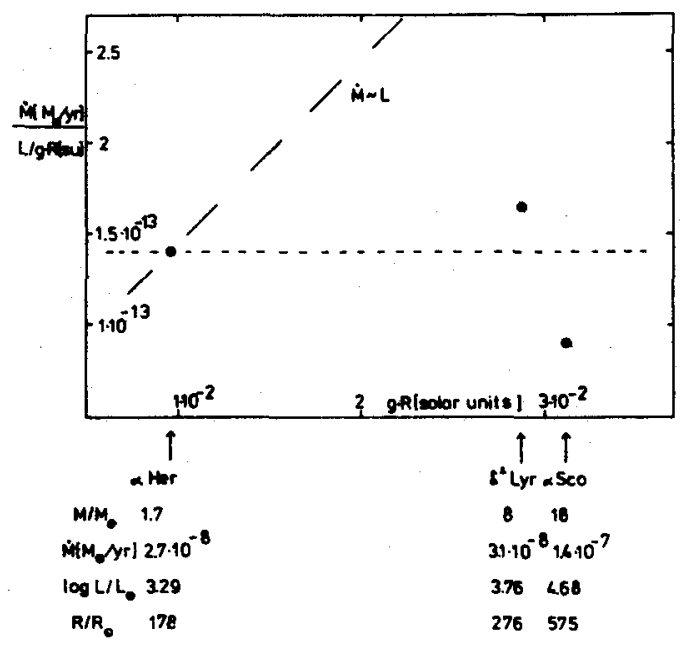

Fig. 6 New absolute calibration of mass-loss relation dM/dt $\sim \mathrm{L} / \mathrm{g} . \mathrm{R}$.

VII. Stellar Winds: from M Supergiants over K Glants to the Sun

As mentioned already (Fig. 1), there is clear observational evidence that all cool stars lose mass, and stellar winds in cool stars seem to have a common origin in non-equilibrium processes (e.g., shock waves) that arise because of the outer convective zones.

The sun is known to be losing mass at a mean rate of $2.2 \cdot 10^{-14}$ $M_{\theta} / y \mathbf{r}$ (Hundhausen, 1972). A daring extrapolation of the red giant mass-loss relation to the sun would predict a factor of $\sim 6$ more. For the solar wind, the ratio of kinetic wind energy $E_{k i n}$ to the potential energy $E_{\text {pot }}$ required to remove matter from the sun is $E_{k i n} / E_{\text {pot }}=1 / 2$, while in red giants' slowly expanding shells there is always $E_{\text {pot }}>E_{k i n}$. Therefore, the fraction of the solar luminosity spent for the solar wind is lower by only a factor of 4 than in 


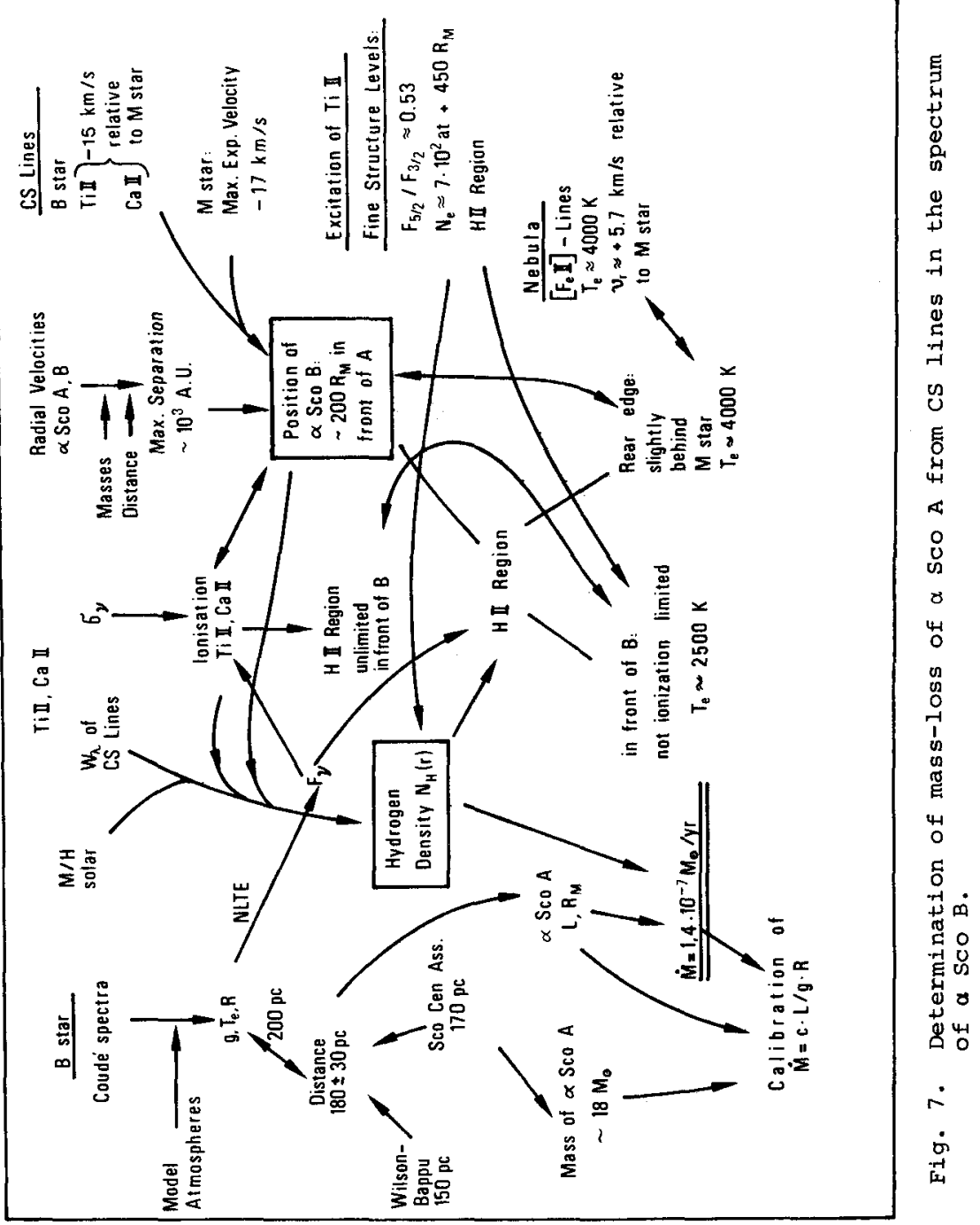


M giants and supergiants, where it is $\sim 2.5 \cdot 10^{-6}$.

Furthermore, the solar wind velocity is just what one would expect from extrapolating red giant stellar wind velocities $\left(v_{w} \sim v_{\text {esc }}{ }^{2}\right)$. Physically this means that the ratio of the kinetic energy carried by the stellar winds to the potential energy required to remove matter from the stars increases from supergiants over giants to the sun by a factor of $\sim 10$.

It appears likely, that the origin of this behaviour is a continuous decrease of the maximum temperature which can be maintained at the base of the flow from the sun over giants to supergiants. Parker's (1963) simple scaling formula predicts $T_{c o r} \sim \mathrm{g} R$. If applied to red giants, we find $\mathrm{T}_{\mathrm{cor}} \leq 2 \cdot 10^{5} \mathrm{~K}$ for the KO III giant B Gem, while $2.610^{5}$ has been observed (Gerola et al., 1975); and for $\alpha{ }^{1}$ Her, Parker's formula predicts $T=13000 \mathrm{~K}$, while the chromospheric temperature of $\alpha{ }^{1}$ Her must be below 20 o00 K since the HeI line $10832 \&$ is not visible. The presence of HeI $10832 \&$ is an indicator of chromospheric regions hotter than $\sim 20000 \mathrm{~K}$ (Vaughan and Zirin, 1968). If $T_{\text {cor }} \sim g \cdot R$ and $v_{w} \sim g \cdot R$, only giants with wind velocities of more than $\sim 30 \mathrm{~km} / \mathrm{s}$ should reveal the HeI line. This is essentially (not strictly) valid, since a number of KII giants, $G$ and $K$ supergiants $\left(30 \mathrm{~km} / \mathrm{s} \leq v_{W} \leq 75 \mathrm{~km} / \mathrm{s}\right)$ do show the HeI line, while $M$ giants and supergiants usually do not.

One should also mention that chromospheric scale helghts (caused by turbulent motions) increase steeply with decreasing gravity and approach the order of stellar radii in bright supergiants.

In summary, the above arguments make us believe that concerning wind velocity, efficiency of energy transmission to the winds (fraction of luminosity used for winds), kinetic wind energies and coronal (chromospheric) temperatures, there is a qualitative relationship between all cool stars. Suppose we would live near an A type star. Present observations of red giants alone would allow us to predict roughly the coronal temperature as well as the velocity and density (too high by a factor of $\sim 4$ ) of the stellar wind of a $\mathrm{G} 2 \mathrm{~V}$ star.

\section{Mass-Loss and Stellar Evolution}

Ever since astronomers tried to understand late evolutionary stages and the final fate of stars, mass-loss in advanced stages of evolution has been a missing link. Recent progress seems promissing, 
though we may be far from a quantitative solution of the mass-loss problem.

As a first step, several authors have combined the mass-loss relation in the form $\mathrm{dM} / \mathrm{dt}=\eta^{4} 10^{-13} \mathrm{~L} / \mathrm{g} \mathrm{R}$ with stellar evolution calculations (Fusi-Pecci and Renzini, 1976; Mengel, 1976; Wood and Cahn, 1977). The factor $\eta$ was introduced as an adjustable parameter of the order of unity because of the uncertainty of mass-loss rates. Comparison with properties of stars in advanced stages of stellar evolution - in principle, all information about red giant mass-loss is contained in cluster HR-diagrams - enables us then to impose restrictions on $\eta$, as the following examples show:

1. The presence of very blue horizontal branch (HB) stars in metal poor clusters requires masses less than $0.55 \mathrm{M}_{0}$ and a mass-loss of $\sim 0.2 \mathrm{M}_{\ominus}$ on the red giant branch preceding the HB phase. This requirement yields $n=0.35$ (Renzini, 1977, and literature quoted therein).

The absence of very bright asymptotic giant branch stars (double shell burning phase) in metal poor globular clusters requires additional loss of $0.1 \mathrm{M}_{0}$ on the AGB. This leads to $\eta=0.40$ (Renzini 1977). So, not unexpected, for both giant branches the same massloss efficiency seems to be required.

2. For metal rich clusters (like 47 Tuc), Gingold (1977) has shown that the red horizontal branches require $n \leq 0.5$.

3. Wood and Cahn (1977) have proposed that the observed period distribution of Mira stars, in particular the existence of highvelocity and Halo Miras with periods less than 300 days, can be understood if mass-1oss with $n$ between 0.25 and 0.50 is taken into account.

4. A less stringent limit is imposed by the (poorly known) upper mass limit $M_{W D}$ of stars still ending up as white dwarfs. Tinsley (1975) has summarized evidence for $M_{W D}=(4 \pm 1) M_{0}$ which would require $\eta=0.58$ (Renzini, 1977).

In summary, the study of the evolution of low-mass stars (metalrich and -poor) leads to $n=0.4 \pm 0.1$. The near coincidence with the revised direct empirical scale $(\eta=0.35 \pm 0.2)$ gives us some confidence in further applications of the simple dimensional formula.

In particular, it appears reasonable to extrapolate the formula to the short evolutionary phases near the tips of the giant branches 
where most of the total mass-loss is predicted to occur (besides the planetary nebulae), but where mass-loss has not yet been observed.

The upper mass limit of stars still becoming white dwarfs is then $M_{W D}=3.6 M_{O}$. Probably, this is only a typical value. In individual cases, mass-loss obviously can be more effective (Weidemman, 1977).

Observational evidence (section VII), however, is not in favour of significant mass-loss already on the subgiant or lower giant branch, as has been suggested by Dearborn and Eggleton (1977) to explain the observed ${ }^{12} \mathrm{C} /{ }^{13} \mathrm{C}$ ratios in giants.

Acknowledgements: It is a pleasure to thank the Hale observatories and the California Institute of Technology for repeated guest investigator privileges. The Deutsche Forschungsgemeinschaft supported this work with two travel grants.

\section{References:}

Bernat, A.P., 1977, Ap. J. $\underline{213}, 756$.

Bernat, A.P., Lambert, D.L., 1975, Ap. J. 201, I 153

1976 a, Ap. J. 204, 830

1976 b, Ap. J. 210, 395.

Chiu, H.Y., Adams, P.J., Linsky, J.L., Basr1, G.S., Maran, S.P., Hobbs, R.W., 1977, Ap. J. 211, 453 .

Cohen, J., 1976, Ap. J. 203, I 127 .

Dearborn, D.S.P., Eggleton, P.P., 1976, Q.J.R.A.S. 17, 448 .

Deutsch, A.J., 1956, Ap. J. 123, 210.

1960, in Stellar Atmospheres (ed. J.L. Greenstein),

The University of Chicago Press, Chicago, p. 543.

Eggen, O.J., 1974, Ap. J. 188, 59 .

Faulkner, D.J., 1971, Proc. Astr. Soc. Austr. 2, 26.

Fusi-Pecci, F., Renzini, A., 1976, Astr. \& Astrophys. $46,417$.

Gehrz, R.D., woolf, N.J., 1971, Ap. J. 165, 285.

Gerola, H., Linsky, J.L., Shine, R., Mc Clintock, W., Henry, R.C.,

Moos, H.W., 1974, Ap. J., 193, L 107.

Gillet, F.C., Low, F.J., Stein, W.A., 1968, Ap. J. 154, 677 .

Gingold, R.A., 1977, M.N.R.A.S., 178, 533.

Goldberg, L.. 1976, Mem. Soc. Roy. Sci. Liège $6^{\text {e }}$ Serie 9,387 .

Goldberg, L., Ramsey, L., Testermann, L., Carbon, D., 1975, D.

Ap. J. 199, 427 .

Kudritzki, R.P., Reimers, D. 1977, Astr. \& Astrophys. submitted. 
Mengel, J.G., Astr. \& Astrophys. 48, 83.

Münch, G., Roester, S., Trauger, J., 1976, Carnegie Yearbook, 1975

p. 283 .

Parker, E.N., 1963, Interplanetary Dynamical Processes, Interscience Publ., New York.

Reimers, D., 1973, Astr. \& Astrophys. 24, 79.

1975 a, Mem. Soc. Roy. Sci. Liège, $6^{\mathrm{e}}$ Serie $\underline{8}, 369$.

$1975 \mathrm{~b}$, in Problems in Stellar Atmospheres and Envelopes

ed. B. Baschek, W.H. Kegel, G. Traving, Springer-Verlag, Berlin-

Heidelberg - New York p. 229.

Reimers, D., 1977 a, Astr. \& Astrophys. 54, 485.

1977 b, Astr. \& Astrophys. 57, 395.

1977 C, Astr. \& Astrophys. 61, 217.

Renzini, A., 1977, in Lectures at the 7 th Advanced Course Saas-Fee

"Advanced Stages in Stellar Evolution".

Saito, M., 1970, Publ. Astr. Soc. Japan, 22, 455.

Sanner, F., 1976, Ap. J. Suppl. 32, 115.

Serkowski, K., 1966, Ap. J. 144, 857 .

Struve, 0., Zebergs, v., 1962, Astronomy of the 20th Century,

MacMillan Comp., New York, p. 303.

Tinsley, B.A., 1975, P.A.S.P. $87,837$.

Vaughan, A., Zirin, H., 1968, A.p. J. 152, 123.

Weidemann, V., 1977, Astr. \& Astrophys. 59, 411.

Weymann, R., 1962, Ap. J. 136, 844 .

Wilson, W.J., Barrett, A.H., 1972, Astr. \& Astrophys. 17, 385.

Wood, P.R., Cahn, J.H., 1977, Ap. J., 211, 499.

Woolf, N.J., Ney, E.P., 1969, Ap. J. 155, L 181.

DISCUSSION Of paper by REIMERS:

KIPPENHAHN: You try to estimate the mass loss of quite a wide range of stars including the sun with the same formula and you suggest by doing this that the same mechanism is responsible for mass loss. Since radiation pressure is negligible for the sun, do you believe that it is also not important for other stars which follow your formula?

REIMERS: I think that radiation pressure on grains is not the mechanism for giants and supergiants up to say, $\alpha$ ori. In the most luminous and cool stars with thick dust shells this process may become effective (as the increase of $\mathrm{OH}$ - maser velocities with infrared excess seems to indicate). The presence of massloss in stars like HD 221170 with $(\mathrm{Fe} / \mathrm{H}) \approx-2.7$ seems to exclude the dust pressure mechanism in giants. 
BIERMANN: What is the dependence of the upper mass limit for white dwarfs on the constant c?

RENZINI: It is a roughly linear relation - we found $M_{w d} \approx 1.3 \mathrm{c}$
$\quad+3.4$.

APPENZELLER: As has been noted in the discussion, hot stars also loose mass. Could this have any influence on your analysis in cases where you derive the outflow from a late-type star from the spectrum of the hot companion?

REIMERS: No. The possible mass-loss rate of aSco $B$ is too small to have any influence on the massive wind from the $M$ supergiant.

SwINGS: I simply wish to point out that Drs. Gilra, snow and I have obtained Copernicus spectra of the companion of Antares in order to study the material ejected by the $M$ supergiant seen in absorption in front of the B star. The study of the data has just begun. 